\title{
On Least Squares Solutions of Matrix Equation $\mathrm{MZN}=\mathrm{S}$
}

\author{
Yuping Zhang, Changzhou Dong, Jianmin Song \\ School of Mathematics and Science, Shijiazhuang University of Economics, Shijiazhuang, China \\ Email: dongchangzh@sina.com, yuping.zh@163.com
}

Received September 29, 2013; revised October 31, 2013; accepted November 10, 2013

Copyright (C) 2013 Yuping Zhang et al. This is an open access article distributed under the Creative Commons Attribution License, which permits unrestricted use, distribution, and reproduction in any medium, provided the original work is properly cited.

\section{ABSTRACT}

Let $P$ be a given Hermitian matrix satisfying $P^{2}=I$. Using the eigenvalue decomposition of $P$, we consider the least squares solutions to the matrix equation $M Z N=S$ with the constraint $P Z=Z P$.

Keywords: Matrix Equation; Eigenvalue Decomposition; Canonical Correlation Decomposition; Reflexive Matrix; Least Squares Solution

\section{Introduction}

Throughout we denote the complex $m \times n$ matrix space by $\mathbb{C}^{m \times n}$. The symbols $I, M^{*}, M^{-1}$ and $\|M\|$ stand for the identity matrix with the appropriate size, the conjugate transpose, the inverse, and the Frobenius norm of $M \in \mathbb{C}^{m \times n}$, respectively.

The reflexive matrices have extensive applications in engineering and scientific computation. It is a very active research topic to investigate the reflexive solution to the linear matrix equation

$$
M Z N=S
$$

where $M$ and $N$ are given matrices. For instance, Cvetković-Ilić [1] and Peng et al. [2] have given the necessary and sufficient conditions for the existence and the expressions of the reflexive solutions for the matrix Equation (1) by using the structure properties of matrices in required subset of $\mathbb{C}^{m \times n}$ and the generalized singular value decomposition (GSVD); Different from [1,2], Ref. [3] has considered generalized reflexive solutions of the matrix Equation (1); in addition, Herrero and Thome [4] have found the reflexive (with respect to a generalized $\{k+1\}$-reflection matrix $P$ ) solutions of the matrix Equation (1) by the (GSVD) and the lifting technique combined with the Kronecker product.

\section{The Reflexive Least Squares Solutions to Matrix Equation (1)}

We begin this section with the following lemma, which can be deduced from [5].

Lemma 1. (Theorem 3.1 in [5]) Let the canonical cor- relation decomposition of matrix pair $[A, C]$ and $B^{*}, D^{*} \quad$ with $\quad A \in \mathbb{C}^{m \times n}, C \in \mathbb{C}^{m \times k}, B \in \mathbb{C}^{p \times q}, D \in \mathbb{C}^{l \times q}$. rank $A=g, \operatorname{rank} C=h, \operatorname{rank} B=g^{\prime}, \operatorname{rank} D=h^{\prime}$ be given as

$$
\begin{aligned}
& A=U\left(\Sigma_{A} 0\right) E_{A}^{-1}, C=U\left(\Sigma_{C} 0\right) E_{C}^{-1}, \\
& B^{*}=V\left(\Sigma_{B} 0\right) E_{B}^{-1}, D^{*}=V\left(\Sigma_{D} 0\right) E_{D}^{-1},
\end{aligned}
$$

where

$$
\Sigma_{A}=\left(\begin{array}{ccc}
I_{i} & & \\
& \Lambda_{j} & \\
\overline{0} & - & \underline{0} \\
& \Delta_{j} & \\
& & I_{t}
\end{array}\right), \Sigma_{C}=\left(\begin{array}{c}
I_{h} \\
\overline{0}
\end{array}\right)
$$

with the same row partitioning, and $g=i+j+t$,

$$
\begin{aligned}
& \Lambda_{j}=\operatorname{diag}\left(\lambda_{i+1}, \cdots, \lambda_{i+j}\right), 1>\lambda_{i+1} \geq \cdots \geq \lambda_{i+j}>0, \\
& \Delta_{j}=\operatorname{diag}\left(\Delta_{i+1}, \cdots, \Delta_{i+j}\right), 1>\Delta_{i+1} \geq \cdots \geq \Delta_{i+j}>0, \Lambda_{j}^{2}+\Delta_{j}^{2} \\
& =I_{j}, \\
& U=\left(\begin{array}{cccccc}
u_{1} & u_{2} & u_{3} & u_{4} & u_{5} & u_{6} \\
i & j & h-i-j & m-h-j-t & j & t
\end{array}\right), \\
& \Sigma_{B}=\left(\begin{array}{ccc}
I_{i^{\prime}} & & \\
& \bar{\Lambda}_{j^{\prime}} & \\
\overline{0} & - & \underline{0} \\
& \bar{\Delta}_{j^{\prime}} & \\
& & I_{t^{\prime}}
\end{array}\right), \Sigma_{C}=\left(\begin{array}{c}
I_{h^{\prime}} \\
\overline{0}
\end{array}\right)
\end{aligned}
$$


with the same row partitioning, and $g^{\prime}=i^{\prime}+j^{\prime}+t^{\prime}$,

$$
\bar{\Lambda}_{j^{\prime}}=\operatorname{diag}\left(\bar{\lambda}_{i^{\prime}+1}, \cdots, \bar{\lambda}_{i^{\prime}+j^{\prime}}\right), 1>\bar{\lambda}_{i^{\prime}+1} \geq \cdots \geq \bar{\lambda}_{i^{\prime}+j^{\prime}}>0,
$$

$$
\bar{\Delta}_{j^{\prime}}=\operatorname{diag}\left(\bar{\Delta}_{i^{\prime}+1}, \cdots, \bar{\Delta}_{i^{\prime}+j^{\prime}}\right), 1>\bar{\Delta}_{i^{\prime}+1} \geq \cdots \geq \bar{\Delta}_{i^{\prime}+j^{\prime}}>0, \bar{\Lambda}_{j^{\prime}}^{2}+\bar{\Delta}_{j^{\prime}}^{2}=I_{j^{\prime}},
$$

$$
V=\left(\begin{array}{cccccc}
v_{1} & v_{2} & v_{3} & v_{4} & v_{5} & v_{6} \\
i^{\prime} & j^{\prime} & h^{\prime}-i^{\prime}-j^{\prime} & q-h^{\prime}-j^{\prime}-t^{\prime} & j^{\prime} & t^{\prime}
\end{array}\right),
$$

and let

$$
U^{*} F U=\left(F_{i j}\right), F_{i j}=u_{i}^{*} F u_{j}, i, j,=1, \cdots, 6 .
$$

Then general forms of least squares solutions $X, Y$ of matrix equation

$$
A X B+C Y D=F
$$

are as follows:

$$
\begin{array}{r}
X=E_{A}\left(\begin{array}{cccc}
F_{11}-R & F_{15} \bar{\Delta}^{-1} & F_{16} & X_{14} \\
\Delta^{-1} F_{51} & X_{22} & \Lambda F_{26}+\Delta F_{56} & X_{24} \\
F_{61} & F_{62} \bar{\Lambda}+F_{65} \bar{\Delta} & F_{66} & X_{34} \\
X_{41} & X_{42} & X_{43} & X_{44}
\end{array}\right) E_{B}^{*}, \\
Y=E_{C}\left(\begin{array}{cccc}
R & F_{12}-F_{15} \bar{\Delta}^{-1} \bar{\Lambda} & F_{13} & Y_{14} \\
F_{21}-\Lambda \Delta^{-1} F_{51} & Q & F_{23} & Y_{24} \\
F_{31} & F_{32} & F_{33} & Y_{34} \\
Y_{41} & Y_{42} & Y_{43} & Y_{44}
\end{array}\right) E_{D}^{*},
\end{array}
$$

where

and

$$
X_{22}=\Lambda\left(F_{22}-Q\right) \bar{\Lambda}+\Lambda F_{25} \bar{\Delta}+\Delta F_{52} \bar{\Lambda}+\Delta F_{52} \bar{\Delta}
$$

$Q, R, X_{14}, X_{24}, X_{34}, X_{44}, X_{41}, X_{42}, X_{43}, Y_{14}, Y_{24}$,

$$
Y_{34}, Y_{44}, Y_{41}, Y_{42}, Y_{43}
$$

are arbitrary matrices.

Theorem 2. Given $M \in \mathbb{C}^{m \times(n+k)}, \quad N \in \mathbb{C}^{(n+k) \times q}$, $S \in \mathbb{C}^{m \times q}$. Then the reflexive least squares solutions to the matrix Equation (1) can be expressed as

$$
\begin{aligned}
& Z=T\left(\begin{array}{cc}
X & 0 \\
0 & Y
\end{array}\right) T^{*}, \\
& X=E_{A}\left(\begin{array}{cccc}
F_{11}-R & F_{15} \bar{\Delta}^{-1} & F_{16} & X_{14} \\
\Delta^{-1} F_{51} & X_{22} & \Lambda F_{26}+\Delta F_{56} & X_{24} \\
F_{61} & F_{62} \bar{\Lambda}+F_{65} \bar{\Delta} & F_{66} & X_{34} \\
X_{41} & X_{42} & X_{43} & X_{44}
\end{array}\right) E_{B}^{*}, \\
& Y=E_{C}\left(\begin{array}{cccc}
R & F_{12}-F_{15} \bar{\Delta}^{-1} \bar{\Lambda} & F_{13} & Y_{14} \\
F_{21}-\Lambda \Delta^{-1} F_{51} & Q & F_{23} & Y_{24} \\
F_{31} & F_{32} & F_{33} & Y_{34} \\
Y_{41} & Y_{42} & Y_{43} & Y_{44}
\end{array}\right) E_{D}^{*},
\end{aligned}
$$

where

$$
X_{22}=\Lambda\left(F_{22}-Q\right) \bar{\Lambda}+\Lambda F_{25} \bar{\Delta}+\Delta F_{52} \bar{\Lambda}+\Delta F_{52} \bar{\Delta}
$$

and

$$
\begin{aligned}
& Q, R, X_{14}, X_{24}, X_{34}, X_{44}, X_{41}, X_{42}, X_{43}, Y_{14}, Y_{24}, \\
& Y_{34}, Y_{44}, Y_{41}, Y_{42}, Y_{43}
\end{aligned}
$$

are arbitrary matrices.

Proof. It is required to transform the constrained problem to unconstrained ones. To this end, let

$$
P=T \operatorname{diag}\left(I_{n},-I_{k}\right) T^{*}
$$

be the eigenvalue decomposition of the Hermitian matrix $P$ with unitary matrix $T$. Obviously, $P Z=Z P$ holds if and only if

$$
\operatorname{diag}\left(I_{n},-I_{k}\right) \bar{Z}=\bar{Z} \operatorname{diag}\left(I_{n},-I_{k}\right)
$$

where $\bar{Z}=T^{*} Z T$. Partitioning

$$
\bar{Z}=\left(\begin{array}{ll}
Z_{11} & Z_{12} \\
Z_{21} & Z_{22}
\end{array}\right), Z_{11} \in \mathbb{C}^{n \times n}, Z_{22} \in \mathbb{C}^{k \times k},
$$

(3) is equivalent to

$$
Z_{12}=-Z_{12}, Z_{21}=-Z_{21}
$$

Therefore,

$$
Z=T \operatorname{diag}(X, Y) T^{*}, X \in \mathbb{C}^{n \times n}, Y \in \mathbb{C}^{k \times k} .
$$

Partition $T=\left(T_{1}, T_{2}\right)$ and denote

$$
A=M T_{1}, C=M T_{2}, B=T_{1}^{*} N, D=T_{2}^{*} N, F=S .
$$

According to (4), (5) and the unitary invariance of Frobenius norm

$$
\begin{aligned}
\|M Z N-S\| & =\left\|M T\left(\begin{array}{cc}
X & 0 \\
0 & Y
\end{array}\right) T^{*} N-S\right\| \\
& =\|A X B+C Y D-F\| .
\end{aligned}
$$

Applying Lemma 2.1, we derive the reflexive least squares solutions to matrix Equation (1) with the constraint $P Z=Z P$ which can be expressed as (2).

\section{Acknowledgements}

This research was supported by the Natural Science Foundation of Hebei province (A2012403013), the Natural Science Foundation of Hebei province (A2012205028) and the Education Department Foundation of Hebei province (Z2013110).

\section{REFERENCES}

[1] D. S. Cvetković-Ilić, "The Reflexive Solutions of the Matrix Equation $A X B=C$," Computers \& Mathematics with Applications, Vol. 51, 2006, pp. 897-902. 
http://dx.doi.org/10.1016/j.camwa.2005.11.032

[2] X. Y. Peng, X. Y. Hu and L. Zhang, "The Reflexive and Anti-Reflexive Solutions of the Matrix Equation $A^{H} X B=C$," The Journal of Computational and Applied Mathematics, Vol. 200, 2007, pp. 749-760. http://dx.doi.org/10.1016/j.cam.2006.01.024

[3] Y. X. Yuan and H. Dai, "Generalized Reflexive Solutions of the Matrix Equation $A X B=D$ and an Associated Optimal Approximation Problem," Computers \& Mathematics with Applications, Vol. 56, 2008, pp. 1643-1649. http://dx.doi.org/10.1016/j.camwa.2008.03.015

[4] A. Herrero and N. Thome, "Using the GSVD and the Lifting Technique to Find $\{P, k+1\}$ Reflexive and AntiReflexive Solutions of $A X B=C$," Applied Mathematics
Letters, Vol. 24, 2011, pp. 1130-1141. http://dx.doi.org/10.1016/j.aml.2011.01.039

[5] G. P. Xu, M. S. Wei and D. S. Zheng, "On Solutions of Matrix Equation $A X B+C Y D=F$," Linear Algebra and Its Applications, Vol. 279, 1998, pp. 93-100. http://dx.doi.org/10.1016/S0024-3795(97)10099-4 